\title{
Untersuchungen an Plattfischen und Nordseegarnelen (Crangon crangon) im Eulitoral des Wattenmeeres nach dem Übergang zum Bodenleben
}

\author{
R. Berghahn \\ Institut für Hydrobiologie und Fischereiwissenschaft der Universität Hamburg; \\ Zeiseweg 9, D-2000 Hamburg 50, Bundesrepublik Deutschland
}

\begin{abstract}
Studies on flatfish and brown shrimp (Crangon crangon) from tidal flats of the Wadden Sea following their transition to a bottom-dwelling mode of life. In spring and early summer, Pleuronectes platessa, Platichthys flesus, Solea solea, and Crangon crangon transform to benthic postlarvae in the shallow water region of the North Frisian Wadden Sea (eastern North Sea). During the first weeks after metamorphosis, they remain in the puddles and drainage gulleys on the tidal flats that are exposed during low tide. Positive rheotaxis prompting the juveniles to swim against the ebb tide currents is an important mechanism for maintaining their position on the flats. When sunlight intensity increases, they continue their locomotory and feeding activity, even during daytime, in the pools still present at low tide. On warm, cloudless days, temperature levels in these pools may increase to lethal thresholds. Consequently, the animals attempt to escape from higher tidal flats near the shore through the drainage gulleys. Such migrations bring about changes in their distribution patterns. Plaice can scarcely be found in near-shore areas, and they are absent at both high and low tide. The responses observed were correlated to physical and chemical data recorded during the observation period. The ecological significance of tidal pools as habitats is discussed.
\end{abstract}

\section{EINLEITUNG}

Auf den tidenperiodisch trockenfallenden Gebieten (Platen) des Wattenmeeres bleiben bei Niedrigwasser zahlreiche flache Pfützen zurück. Sie sind in vielen Fällen nicht größer als der Trichter eines Pierwurms (Arenicola marina), können aber auch zusammenhängende Flächen von mehreren $100 \mathrm{~m}^{2}$ umfassen. Häufig speisen sie kleine Abläufe, die das Wasser in die tieferliegenden Priele leiten.

Derartige Restwasser sind vorübergehend ein wichtiger Lebensraum für Schollen (Pleuronectes platessa L.), Flundern (Platichthys flesus L.) und Garnelen (Crangon crangon L.) (Plagmann, 1937; Linke, 1939; Bergman et al., 1976; Janssen \& Kuipers, 1980). Während und nach der Metamorphose sind die jüngsten Stadien hier von ihren älteren Artgenossen und anderen potentiellen aquatischen Freßfeinden weitgehend räumlich getrennt. Erst später gehen die Jungtiere dazu über, Nahrungswanderungen im Tidenrhythmus von den Prielen auf die Platen und zurück durchzuführen, bei denen sie die hochgelegenen, ufernahen Platen allerdings kaum noch erreichen. Schollen beginnen etwa 4 Wochen nach der Metamorphose mit der Tidenwanderung (Bergman et al., 1976). In dieser Zeit ist ein starker Rückgang der Abundanzen zu verzeichnen, den 
die Autoren darauf zurückführen, daß die Schollen in den Einflußbereich großer Individuen von Crangon crangon und Carcinus maenas gelangen und ein beträchtlicher Teil gefressen wird. Für $C$. crangon ist der Zeitpunkt des Ubergangs zur Tidenwanderung nach Kuipers \& Dapper (1981) spätestens bei einer Länge von $3,5 \mathrm{~cm}$ erreicht.

In den ersten Wochen nach der Metamorphose wird allgemein eine erhöhte Sterblichkeit bei Plattfischen vermutet (Rauck \& Zijlstra, 1978). Im Rahmen einer sich über 4 Jahre erstreckenden Untersuchung zu der Frage, in welchem Maße intra- und interspezifische Konkurrenz sowie Räuber-Beute-Beziehungen auf diesen Sterblichkeitsverlauf Einfluß nehmen, konnten zahlreiche Beobachtungen zum Verhalten dieser Wattenbewohner angestellt werden. Im Zusammenhang damit wurde auch die Bedeutung verschiedener abiotischer Faktoren für den Sterblichkeitsverlauf nach der Metamorphose beleuchtet, wobei die Verbindung von fischereibiologischen Methoden mit Freilandbeobachtungen zum Verhalten der Tiere und synchronen wasserchemischen Insitu-Messungen umfassendere Aussagen ermöglichte.

\section{MATERIAL UND METHODEN}

\section{Untersuchungsgebiet}

Das Untersuchungsgebiet liegt im Nordfriesischen Wattenmeer zwischen der Hamburger Hallig und der Insel Nordstrand (Abb. 1). Süßwasserzuläufe von Land gestalten die Salzgehaltsverhältnisse vor allem im Frühjahr und im Herbst eines jeden Jahres sehr wechselhaft. Der mittlere Tidenhub beträgt $3,5 \mathrm{~m}$.

An 9 Stationen wurden in den Jahren 1979-1982 von März bis November, im Frühjahr im Abstand von 2, später im Abstand von 4 Wochen, die Pfützen und Drainierpriele küstennaher Sand-, Misch- und Schlickwatten untersucht. Die Trockenfallzeiten dieser Gebiete liegen an den Stationen 2, 3, 6, 7 und 8 über 8 Stunden. Die Abläufe führen während dieser Zeit ständig Wasser. Im folgenden sind die wesentlichen Charakteristika der Stationen stichwortartig aufgelistet.

St at i o n 1: hochliegende, "küstenferne", prielferne Arenicola-Misch-Sand-Watten ohne Süßwasserzulauf; nur 1982 regelmäßig aufgesucht; S t a ti o n 2: Schlickwatt im hochliegenden, prielfernen Lahnungsbereich; im Frühjahr und Herbst bei Winden aus W bis NW stark süßwasserbeeinflußt; ab 1979 gelegentlich aufgesucht; $\mathrm{S}$ t a ti on 3 : hochliegende, küstennahe, prielnahe Mischwatten im Lahnungsbereich; Süßwasserzulauf $_{;}$ab 1979 regelmäßig aufgesucht; Station $4:$ Kolk; tiefliegende, prielnahe, süßwasserbeeinflußte Erosions-Mischwatten mit Haupt- und Nebenpriel; nur 1982 regelmäßige Station; Statio n 5: Misch- und Schlickwatten an einem landnahen, verlandenden Prielsystem ohne Süßwasserzulauf; nur 1982 gelegentlich aufgesucht; Station 6: hochliegende Sandwatten; nur 1982 gelegentlich aufgesucht; Station 7 : hochliegender Lahnungsbereich mit vorgelagerten Sandwatten; ab 1979 gelegentliche Station; Station 8: hochliegende Zostera-Arenicola-Watten im Lahnungsbereich, Nordlage; ohne Süßwassereinfluß; ab 1979 regelmäßig aufgesucht; $S$ t a t i o n 9 : Muschelbankkolk direkt an einer ehemaligen Wasserscheide; starke Strömung; Nordlage; nur 1982 regelmäßig aufgesucht. 


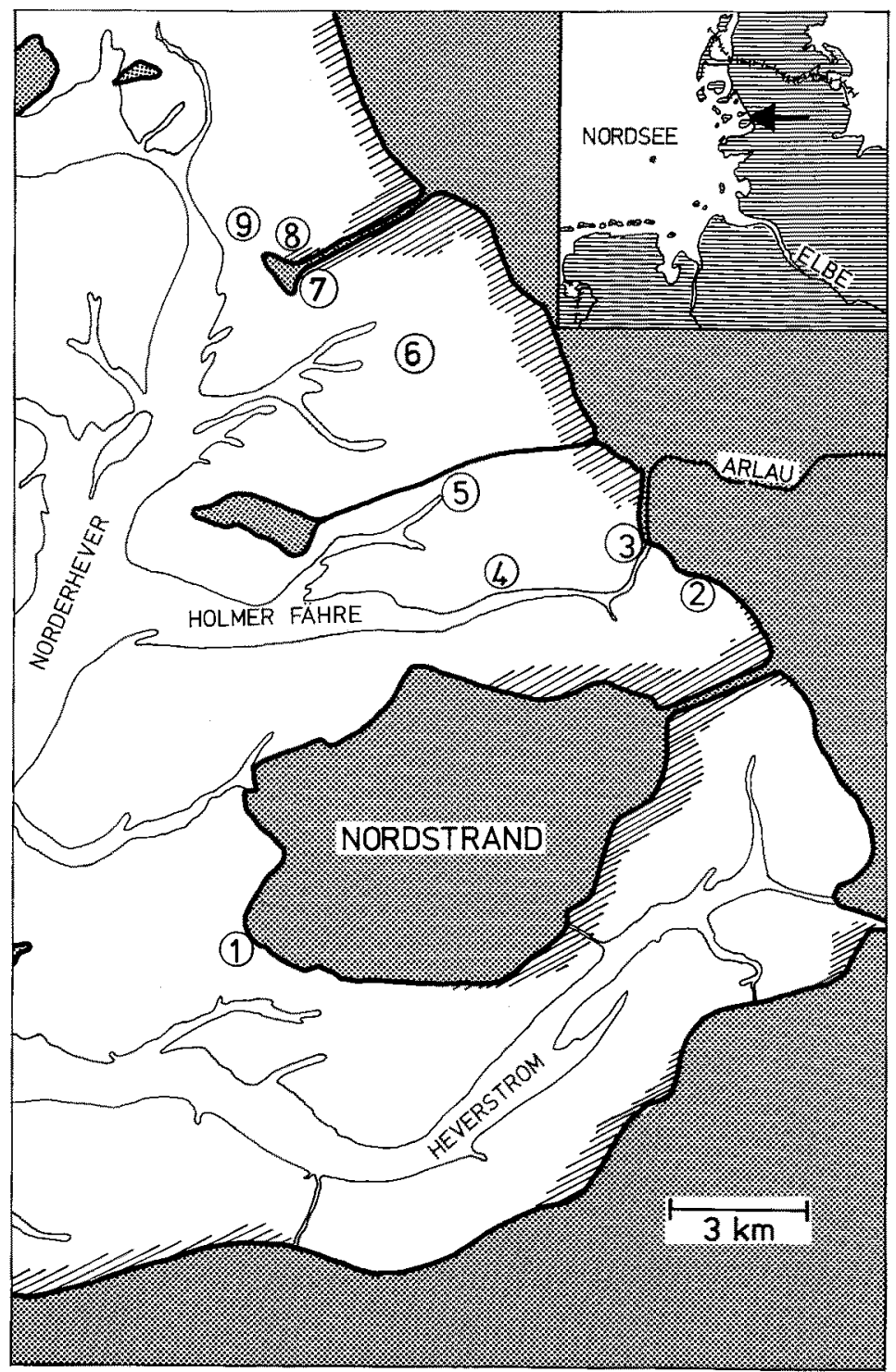

Abb. 1. Untersuchungsgebiet mit Probennahme-Stationen (vgl. Text)

\section{Untersuchungsmethoden}

Die vorliegenden Ergebnisse stützen sich überwiegend auf direkte Beobachtungen, die an 158 Terminen bei jedem Wetter zwischen Tag- und Nachtanbruch vom Zeitpunkt vor der Ausbildung der Pfützen bis zum erneuten Ansteigen des Wassers gemacht werden konnten. Die tägliche Beobachtungsdauer betrug 2-10 Std.

Vor jeder Untersuchung wurden stets in einem unmittelbar benachbarten Restwasser mehrfach die Individuendichten der genannten Arten für vergleichbare Wassertiefen 
ermittelt, indem Sedimentflächen von $10 \times 10 \mathrm{~cm}$ mit dem Finger überstrichen und die aufgeschreckten Tiere gezählt wurden. Diese Werte sowie das Erfassen einer Reihe von Umweltcharakteristika bildeten die Grundlage für die Schätzungen des prozentualen Anteils umherschreitender bzw. -schwimmender Tiere, bezogen auf die Gesamtdichte in den Beobachtungsfeldern, in denen erst nach Abschluß jeder Beobachtungsreihe Störungen der Tiere durch direkte Kontrollzählungen verursacht werden durften. Alle Zählungen und Beobachtungen wie auch die tiden- und wetterbedingten Veränderungen (z. B. Beschattung des Beobachtungsfeldes durch Wolken) wurden mit Zeitangabe sofort handschriftlich protokolliert, in besonderen Fällen auf ein Diktiergerät gesprochen.

Es zeigte sich, daß kleinste Erschütterungen, Beschattungen und schnelle Bewegungen das Verhalten der Tiere ganz wesentlich beeinflussen. Aus diesem Grunde war es bei der Beobachtung in ca. $30 \mathrm{~cm}$ Entfernung von der Wasseroberfläche wichtig, daß der Beobachter zunächst langsam und vorsichtig am Rand der Pfützen niederkniete, die von seinem Körper nicht beschattet werden durften, und 1-2 Min. bewegungslos wartete, bis sich die Tiere wieder aus dem Sediment hervorwagten.

Ergänzend wurden an 150 Tagen insgesamt über 700 Hochwasserfänge und Pfützenbefischungen mit einem Schiebehamen nach Riley (1971) durchgeführt. Das Netz hatte 1,4 mm Maschenweite (von Knotenmitte zu Knotenmitte) und eine Bleileine von $150 \mathrm{~g} / \mathrm{m}$ als Grundkette. Je nach Beschaffenheit des Untergrundes wurden eine ebensolche Bleileine oder 2 schwere Ketten $(30 \times 6 \mathrm{~mm})$ als Scheuchketten eingesetzt. In Wassertiefen über 1,0 m kam in den Jahren 1979, 1981 und 1982 bei 106 Ausfahrten über 1000 mal eine 2-m-Baumkurre mit knotenlosem Nylonnetz von $5 \mathrm{~mm}$ Maschenweite (Kuipers, 1975) zum Einsatz, die von einem flachgehenden Boot mit Außenbordmotor gezogen wurde. Gekurrt wurde - mit Ausnahme gezielt durchgeführter Prielbefischungen bei Niedrigwasser - stets im Zeitraum 1,5 Std. vor bis 1,5 Std. nach Hochwasser. Bei einer Schleppzeit von $3 \mathrm{Min}$. und einer Geschwindigkeit von ca. $1 \mathrm{kn}$ betrug die mit dem Strom abgeschleppte Fläche etwa $200 \mathrm{~m}^{2}$. Die genaue Bestimmung der Hollänge erfolgte mit Hilfe eines an der Kurre montierten Zählrades.

Bei allen Probennahmen wurde die Wassertemperatur auf $1 / 5{ }^{\circ} \mathrm{C}$ genau gemessen. Der Salzgehalt wurde mit einem Refraktometer und die Strömungsgeschwindigkeit mit Treibkörper und Stoppuhr bestimmt. Die Bewölkung wurde in 8, die Sicht in 3 Stufen geschätzt, die Windstärke mit einem Anemo-Handwindmesser ermittelt. An ausgewählten Tagen wurden in Pfützen und Abläufen zusätzlich folgende Meßgrößen gewonnen: (a) Oberflächentemperatur des Sediments (Gulton Tastotherm D 100), (b) Sauerstoffgehalt des Wassers (WTW Oxysonde 57), (c) pH-Wert des Wassers (Knick-Sonde Portacon 652 mit V 456-KNZ), (d) HS ${ }^{-}$-Gehalt des Wassers (colorimetrisch mit Methylenblau, Merck 14416), (e) $\mathrm{NO}_{2}{ }^{-}$-Gehalt des Wassers (colorimetrisch, semiquantitativ).

\section{ERGEBNISSE}

\section{Besiedlung der Pfützen und Abläufe auf den Platen des Wattenmeeres}

$\mathrm{Ab}$ Ende März eines jeden Jahres findet nacheinander bei Schollen, Flundern und Garnelen der Übergang von der planktischen Larvalphase zum Bodenleben statt. Die Dichten in den ufernahen Pfützen lagen für Schollen zwischen 0 und 8 Individuen $/ \mathrm{m}^{2}$, 
für Flundern zwischen 0 und 130 Individuen $/ \mathrm{m}^{2}$ und für Garnelen von 0,5 bis $2 \mathrm{~cm}$ Länge zwischen 0 und mehr als 6000 Individuen $/ \mathrm{m}^{2}$. Stets sind starke orts- und strömungsbedingte Dichteunterschiede, kleinräumige patchiness, die Bevorzugung bestimmter Wassertiefen durch verschiedene Längengruppen in den nur einige Zentimeter tiefen Pfützen sowie starke Veränderungen im Jahresgang zu verzeichnen. Angaben mittlerer Dichten sind daher nur für die punktuellen Untersuchungen sinnvoll. Neben diesen 3 Arten sind in den Pfützen der hochliegenden Misch- und Schlickwatten ab Mitte Juni bis zu 1 Individuum $/ \mathrm{m}^{2}$ gerade metamorphosierter Seezungen (Solea solea) anzutreffen. Im Sommer finden sich darüber hinaus Massen von Corophium volutator (bis zu $400000 /$ $\mathrm{m}^{2}$ ) und Carcinus maenas der 0-Gruppe (bis $\mathrm{zu} 400 / \mathrm{m}^{2}$ ) in und zwischen diesen Restwasserbereichen.

Die Abschätzungen aus den Schiebehamenfängen lassen in Verbindung mit den Ergebnissen aus den Befischungen mit der Baumkurre den Schluß zu, daß der weitaus überwiegende Teil der jüngsten Bodenstadien in den ersten Wochen nach dem Übergang zum Bodenleben sowohl bei Hoch- als auch bei Niedrigwasser auf den hochgelegenen Platen bleibt. In Tabelle 1 sind exemplarisch die Zahlen einer Platenbefischung auf Jungflundern vor einer steilen Uferböschung an Station 3 wiedergegeben: Die Tiere sammeIn sich bei Hochwasser in unmittelbarer Ufernähe, weil hier das Wasser an einer Barriere aufgestaut wird. Diese Tatsache ist für die Gewinnung hoher Abundanzen im relativ kurzen Probennahmezeitraum bei Hochwasser vorteilhaft. Mit dem ablaufenden Wasser werden die Dichten in größerer Entfernung zum Ufer in vergleichbaren Wassertiefen immer geringer, weil die Flundern in den Pfützen und Abläufen bei Ebbe zurückbleiben, wie die Niedrigwasserbefischung zeigt.

Tab. 1. Postlarvendichten $\left(\mathrm{N} / 1000 \mathrm{~m}^{2}\right)$ von Platichthys flesus an Station 3 der Nordstrander Bucht kurz nach Hochstauwasser $(T)$, bei stärker ablaufendem Wasser $(\downarrow)$ und bei Niedrigwasser $(\perp)$ in verschiedenen Entfernungen zur Hochwasserlinie (8. Mai 1981)

\begin{tabular}{|c|c|c|c|c|c|c|}
\hline Zeit & \multicolumn{2}{|c|}{$\begin{array}{l}\text { Ort und Entfemung } \\
\text { zur Uferböschung }\end{array}$} & Tide & $\begin{array}{l}\text { Überstau } \\
{[\mathrm{cm}]}\end{array}$ & $\mathrm{N} / 1000 \mathrm{~m}^{2}$ & $\begin{array}{c}\text { Befischte } \\
\text { Fläche } \\
{\left[\mathrm{m}^{2}\right]}\end{array}$ \\
\hline 6.50 & wasserbedeckte Platen & $20 \mathrm{~m}$ & $T$ & 10 & 1778 & 45 \\
\hline 6.40 & wasserbedeckte Platen & $50 \mathrm{~m}$ & $\mathrm{~T}$ & 30 & 67 & 45 \\
\hline 7.30 & wasserbedeckte Platen & $200 \mathrm{~m}$ & $\downarrow$ & bis 5 & 222 & 45 \\
\hline 8.30 & Ablauf & $100 \mathrm{~m}$ & $\perp$ & $3-5$ & 11667 & 30 \\
\hline 9.00 & Pfütze & $100 \mathrm{~m}$ & $\perp$ & $7-10$ & 13033 & 30 \\
\hline
\end{tabular}

Bei einer Pfützenbedeckung von etwa $10 \%$ der Fläche des 1. Lahnungsfeldes an Station 3 bei Niedrigwasser liegen die zu diesem Zeitpunkt ermittelten Flunderdichten umgerechnet auf die Gesamtfläche bei Hochwasser im Bereich der gefundenen Hochwasserwerte. Tabelle 2 zeigt eine entsprechende Abschätzung für die Individuendichten von Crangon crangon bei Hoch- und Niedrigwasser an Station 3, die Groh (1982) im Jahre 1981 mit demselben Schiebehamen feststellen konnte. 
Tab. 2. Vergleich der aus Pfützenfängen im 1. Lahnungsfeld an Station 3 hochgerechneten Bestandsdichten $\left(\mathrm{N} / \mathrm{m}^{2}\right)$ von Crangon crangon für die Hochwassersituation mit den bei den Hochwasserbefischungen im 1. Lahnungsfeld tatsächlich gefundenen Werten (nach Groh, 1982). Ohne Korrektur auf Netzselektion. NW = Dichten in den Pfützen, HW = Dichten bei Hochwasser $\%$ Pfütze $=$ Anteil der Pfützenbedeckung im 1. Lahnungsfeld, NW' = errechnete Dichten (NW $\frac{\% \text { Pfütze }}{100}$ ) bei Hochwasser

\begin{tabular}{|rcccrr|}
\hline Datum & $\begin{array}{c}\text { Wassertiefe } \\
{[\mathrm{cm}]}\end{array}$ & NW & HW & \% Pfütze & NW $^{\prime}$ \\
\hline 2.7. & 5 & 85 & 39 & 60 & 51 \\
24.7. & $3-4$ & 31 & 21 & 30 & 9 \\
27.8. & 5 & 25 & 15 & 30 & 4 \\
29.8. & $2-3$ & 14 & 6 & 30 \\
\hline
\end{tabular}

Auch in diesen Fällen stimmen die bei Hoch- und Niedrigwasser gewonnenen Zahlen, bezogen auf die zugehörige gesamte Platenfläche bei Hochwasser, größenordnungsmäßig überein. Für Schollen und Seezungen ergaben sich keine widersprechenden Befunde. Allerdings wurde von derartigen Schätzungen aufgrund der relativ geringen Abundanzen abgesehen.

Ubereinstimmend damit zeigten die Fänge mit der 2-m-Baumkurre, daß in jedem Jahr der erste große Schub gerade metamorphosierter Plattfische der jeweiligen Art zwar z. T. massenhaft auf den hochliegenden Platen in Ufernähe, aber nicht oder nur sehr vereinzelt auf den tieferliegenden Platen und bei den Prielbefischungen bei Niedrigwasser gefangen werden konnte. Die Dichten von Crangon crangon $(\leq 1 \mathrm{~cm}$ Länge) waren in Kurrenfängen ohne Korrektur auf Netzselektion auf den hochliegenden Platen des Mischwatts in Ufernähe um den Faktor 10 bis 100 höher als auf den tieferliegenden Platen. Bei den Prielbefischungen bei Niedrigwasser lagen die Dichten noch unter denen von den tiefliegenden Platen.

\section{Bedeutung der abiotischen Faktoren in den Pfützen und Abläufen am Beispiel von Temperatur und Salzgehalt}

Während größere Wasserkörper nur träge auf Veränderungen der Lufttemperatur und der Sonneneinstrahlung reagieren, sind Tiere in Pfützen von wenigen Zentimetern Tiefe sehr hohen Schwankungen der abiotischen Faktoren, vor allem der Temperatur und des Salzgehaltes, ausgesetzt. Eine genaue Darstellung der Meßwerte soll im folgenden nur an ausgewählten, aber charakteristischen Beispielen im Zusammenhang mit synchronen Verhaltensbeobachtungen geschehen, weil insbesondere die Temperatur in den Pfützen einer außerordentlich starken Beeinflussung durch eine Fülle ständig variierender Faktoren unterliegt. Es seien hier nur genannt: (a) Tageslänge, (b) Sonnenstand, (c) Tidenphase in ihrer gegenüber dem Tag/Nacht-Rhythmus versetzten Periodik, (d) Temperatur des Prielwassers, (e) Lufttemperatur, (f) Bewölkungsstärke, ( $g$ ) Bewölkungsdauer, (h) Sicht, (i) Windstärke, (j) Sedimentfärbung, (k) Länge der Trockenfallzeit.

Folgende Extremwerte wurden gemessen: Die ersten Postlarven der Schollen eines Jahrganges müssen im März und im April noch Nachtfröste überstehen. In den 1-3 cm 
flachen Pfützen treten dann Temperaturen unter $0{ }^{\circ} \mathrm{C}$ auf. Mehrfach wiederholte orientierende Versuche mit insgesamt 50 Individuen ergaben, daß die Jugendstadien aus den Pfützen im Gegensatz zur I-Gruppe den Aufenthalt in Wasser von $-1,0$ bis $-1,6^{\circ} \mathrm{C}$ mehrere Stunden in einem der Starre ähnlichen Zustand unbeschadet überstehen (Akklimatisationstemperatur, also aktuelle Oberflächentemperatur des Gewässers: 5,4 bis $8,4^{\circ} \mathrm{C}$; Salzgehalt $\mathrm{S}=25-30$ ). Die später metamorphosierten Individuen können bereits im Mai Temperaturen von $28^{\circ} \mathrm{C}$ exponiert sein. Im Sommer übersteigen die Wassertemperaturen nicht selten $32^{\circ} \mathrm{C}$, wie 1981 in einem und 1982 in 2 Fällen nachgewiesen werden konnte. Die Schwankungen, die für die Tiere im Verlauf eines Tages auftreten, können $15^{\circ} \mathrm{C}$ und mehr betragen.

Die Salzgehaltsverhältnisse sind stark abhängig von den vorherrschenden Windlagen, den Niederschlägen und den resultierenden Abflußmengen aus den Süßwasserzuflüssen. Mit dem auflaufenden Wasser oder bei starken Regenfällen kann der Salzgehalt in den Pfützen innerhalb von Sekunden von $S=20$ auf weit unter $S=10$ absinken, um sich nach weiterer Durchmischung auf einen mittleren Wert einzustellen.

\section{Verhalten der Tiere in Pfützen und Abläufen bei Wassertemperaturen unter $27^{\circ} \mathrm{C}$}

Die folgenden allgemeinen Ausführungen beziehen sich auf Beobachtungen, die im gesamten Untersuchungszeitraum in allen 4 Jahren immer wieder gemacht werden konnten, wobei die Rahmenbedingungen vor allem im Temperaturregime (Akklimatisation, Temperatursteigerung, Extremwerte) in den seltensten Fällen die gleichen waren.

Plattfische und Garnelen schlagen sich bei ablaufendem Wasser in den Pfützen und Abläufen in die obersten Sedimentschichten ein. Bei kühlem oder stürmischem Wetter mit starker Bewölkung bleiben sie im Sediment vergraben. An wärmeren Frühjahrsund Sommertagen kommen abhängig von der Akklimatisationstemperatur, also der aktuellen Temperatur des Küstenwasser, mit steigender Intensität der Sonneneinstrahlung immer mehr Tiere aus dem Sediment hervor, und die Bewegungsaktivität des Einzeltieres wird größer. Die Postlarven der Plattfische und Garnelen schwimmen bzw. schreiten in den Pfützen umher, wenn sie sich nicht gestört fühlen. Sie nehmen Nahrung auf, zunächst vornehmlich Plankton, später kleine Benthosorganismen oder exponierte Teile davon.

In Ergänzung zu Gibson (1980) können im Zusammenhang damit alle Verhaltensweisen, die er an Schollen zwischen 3 und $10 \mathrm{~cm}$ Länge vor der englischen Küste in 1-2 m Wassertiefe studiert hat, schon an gerade metamorphosierten Schollen zwischen 1,0 und 2,6 cm Länge in den Pfützen registriert werden: sporadisches Schwimmen, abrupte Drehungen, Beißen, Kauen, "Gähnen", Aufbäumen und Aufrollen.

Mit tidenbedingt steigendem Wasserspiegel erhöht sich der Anteil aktiver Tiere auf $100 \%$. Sie bewegen sich mit der Strömung. Auch bei ablaufendem Wasser gehen sie zunächst mit der Strömung. Fällt das Wasser schnell, werden sie dabei in der Regel nicht trockenfallen, sondern sich immer aktiv in die angrenzende Pfütze arbeiten.

Es wurden jedoch des öfteren sogar 5 Stunden nach Trockenfallen lebende Plattfische von 0,8 bis $1,2 \mathrm{~cm}$ Länge auf den trockenen Misch- und Schlickwatt-Abschnitten zwischen den Pfützen angetroffen. Sie hatten offenbar der Verdunstung bzw. Versickerung ihrer Pfützenflächen nicht entkommen können. Wenn man sich einer Pfütze nicht 
vorsichtig genug nähert, kann man häufig beobachten, daß die Tiere aus dem Wasser aufs Trockene flüchten. Diese Fluchtreaktion tritt möglicherweise auch dann auf, wenn Vögel die Pfützen durchwaten.

Bei ablaufendem Wasser zeigen die Tiere von einer bestimmten Strömungsgeschwindigkeit (etwa $0,05 \mathrm{~m} / \mathrm{s}$ ) an in Abhängigkeit zur Wassertiefe schlagartig positive Rheotaxis. In Wassertiefen von 0,5 bis $10 \mathrm{~cm}$ konnte beobachtet werden, daß sich ein Teil mit dem Kopf gegen den Wasserstrom gerichtet einschlägt, während eine mit der Intensität der Sonneneinstrahlung zunehmende Anzahl sich stromauf zu bewegen versucht. Dieses Verhalten tritt vor allem in den Pfützenabläufen auf und ist bei Crangon crangon und Jungflundern besonders stark, bei Schollen und Seezungen weniger ausgeprägt.

Die 0,5 bis $1,2 \mathrm{~cm}$ großen Flundern gelangen dabei in Sprüngen von bis zu $20 \mathrm{~cm}$, die von 10-20 Sekunden langen Ruhephasen unterbrochen werden, immer weiter stromaufwärts. Schließlich kommen sie an eine Stelle, an der sie durch stärkere Wasserwirbel wieder ein Stück stromab verdriftet werden, oder aber sie erreichen die den Ablauf speisende Pfütze. Die positive Rheotaxis ist ausschlaggebend dafür, daß sich die Jugendstadien der 4 genannten Arten zunächst in den ufernahen Pfützen und Abläufen konzentrieren. Das Schema in Abb. 2 zeigt, wie auf diese Weise eine gewisse Standortkonstanz garantiert wird.

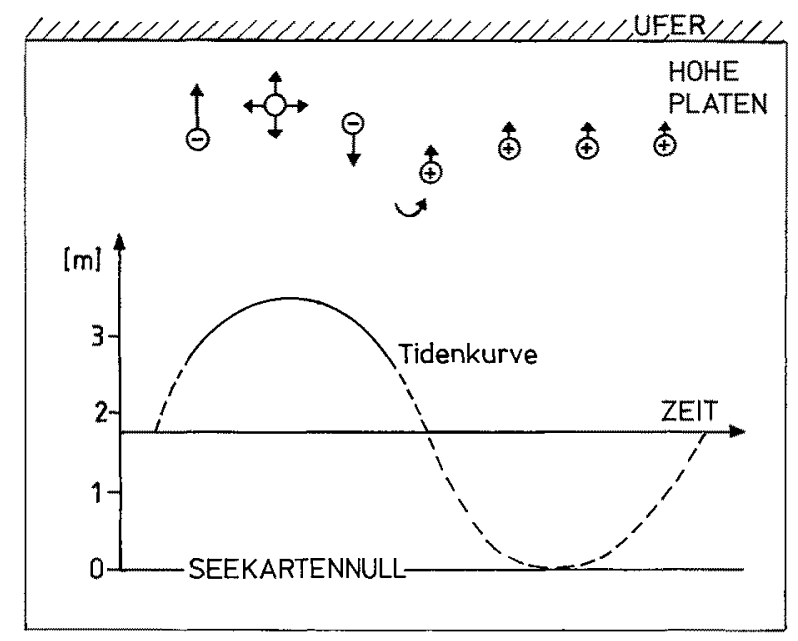

Abb. 2. Schema der Bewegungsrichtungen der Postlarven und Juvenilen von Scholle, Flunder, Seezunge und Garnele auf den hohen Platen im Tidenverlauf. Die durchgezogene Linie der Tidenkurve gibt das Zeitintervall an, in dem die hohen Platen überstaut sind. + Organismus (im Ablauf) mit positiver, - mit negativer Rheotaxis

\section{Verhalten der Tiere in Pfützen und Abläufen bei Wassertemperaturen über $27^{\circ} \mathrm{C}$}

Eine völlige Umkehrung des zuvor beschriebenen Verhaltens tritt bei der gleichen Altersstufe der untersuchten Arten an warmen Frühjahrs- und Sommertagen bei Niedrigwasser auf: Anstatt positiver Rheotaxis beginnen die Schollen, Flundern, Seezungen 
und Garnelen in den Abläufen plötzlich alle, fluchtartig, mit dem Kopf voran, nahe der Wasseroberfläche stromab zu schwimmen. Dieses Phänomen wird im folgenden Exodus genannt. Es konnte 1981 nur zweimal und während des warmen Frühjahrs und Sommers 1982 insgesamt nur fünfmal beobachtet werden, und zwar ausschließlich dann, wenn die Niedrigwasserzeiten so liegen, daß sie an heißen, fast wolkenlosen Tagen mit nur leichten bis schwachen Winden $(0-5,4 \mathrm{~m} / \mathrm{s})$ in Phasen intensiver Sonneneinstrahlung fallen. Corophien und Strandkrabben der 0-Gruppe sind an diesem Exodus praktisch nicht beteiligt.

Ein derartiger Exodus hat in der Regel für die betroffenen jungen Plattfische katastrophale Folgen: Bei anhaltender Sonneneinstrahlung können wiederholt Plattfische gesehen werden, die regungslos in der Strömung trudeln. Am Folgetag eines Exodus traten in den prielnahen Hochwasserfängen über $16 \%$ tote, aber ansonsten unversehrte Schollen auf. Normalerweise sind alle gefangenen Jungschollen sehr vital. Immer wenn der Exodus in größerem Umfang einsetzt, finden sich vor allem Lach- und Silbermöwen (Larus ridibundus und Larus argentatus), die sonst an der Wasserkante des auf- bzw. ablaufenden Wassers oder über die Platen verteilt sind, in großen Mengen in diesen Abläufen versammelt, um die auf der Flucht befindlichen, im Wasser schwimmenden Tiere zu erbeuten. In einem Ablauf von $1 \mathrm{~m}$ Breite stehen dann auf $100 \mathrm{~m}$ bis zu 200 Möwen.

Die Plattfische und Garnelen in Pfützen, die keinen Wasserablauf besitzen, werden, wenn in den Abläufen der Exodus einsetzt, mit zunehmender Sonneneinstrahlung aktiver. Ihre Bewegungen sind hingegen völlig ungerichtet, weil keine Strömung vorhanden ist. Bald darauf beginnen die Plattfische, rastlos nahe der Wasseroberfläche zu schwimmen. Gleichzeitig erhöht sich der prozentuale Anteil aktiver Individuen von Crangon crangon auf $100 \%$. Die Garnelen schreiten hastig, einige schwimmen umher.

In 3 Fällen bot sich die seltene Gelegenheit, die Veränderungen einiger Meßgrößen über mehrere Stunden zu verfolgen, die für die direkte oder indirekte Auslösung eines Exodus theoretisch in Frage kommen können: (a) Wassertemperatur, (b) Salzgehalt des Wassers, (c) Oberflächentemperatur des Sediments, (d) $\mathrm{O}_{2}$-Gehalt des Wassers, (e) $\mathrm{pH}$ Wert des Wassers, (f) $\mathrm{HS}^{-}{ }^{-}$-Gehalt des Wassers, ( $\mathrm{g}$ ) $\mathrm{NO}_{2}{ }^{-}$-Gehalt des Wassers. In Abb. 3 sind exemplarisch die Ergebnisse eines dieser wasserchemischen und -physikalischen Meßprogramme, das an Station 3 an einem warmen Sommertag bei Niedrigwasser durchgeführt wurde, den synchronen biologischen Beobachtungen gegenübergestellt worden. Der untersuchte Ablauf hatte zu Beginn der Probennahme eine Breite von 0,8 m und eine Tiefe von $6 \mathrm{~cm}$. Die Strömungsgeschwindigkeiten lagen zwischen 0,2 und $0,1 \mathrm{~m} / \mathrm{s}$. Der Salzgehalt schwankte zwischen $\mathrm{S}=30$ und $\mathrm{S}=32 . \mathrm{NO}_{2}{ }^{-}$war im Ablauf stets $<0,1 \mathrm{mg} / 1, \mathrm{HS}^{-}$stets $<0,01 \mathrm{mg} / 1$ unabhängig von der Tatsache, ob ein Exodus zu verzeichnen war oder nicht. Wie Abb. $3 \mathrm{~B}$ und $\mathrm{C}$ zeigen, lagen auch die Sauerstoff- und die $\mathrm{pH}$-Werte im Normalbereich. In der danebenliegenden, gleichzeitig untersuchten Pfütze (Abb. $3 \mathrm{E}$ ) von etwa $3 \mathrm{~m}^{2}$ Größe und 1-3 cm Tiefe mit dichtem Bewuchs von Zostera noltii waren Sauerstoffübersättigungen und ein Anstieg des pH-Wertes zu verzeichnen.

Der Vergleich der synchronen Befunde für die Bewölkung, die Crangon-Aktivität und die Wassertemperatur macht deutlich, daß in den Abläufen die Aktivitätssteigerung und der Exodus direkt mit den hohen Temperaturen in den oberen Sedimentschichten, hervorgerufen durch die Sonneneinstrahlung, in Beziehung stehen. Diese Feststellung 


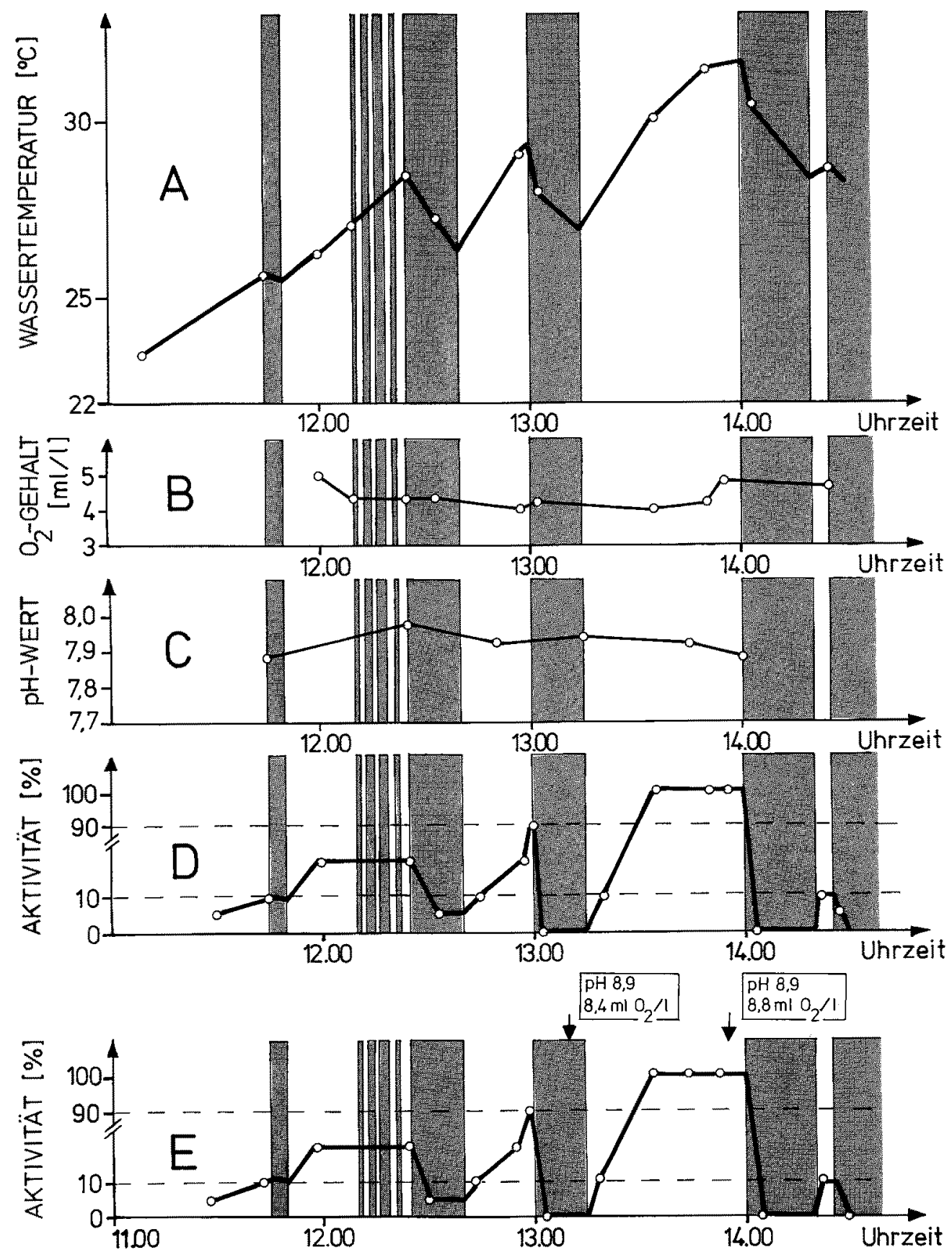

Abb. 3. Zeitlicher Verlauf ausgewählter Meßgrößen synchron zu den Aktivitätsänderungen von Crangon crangon (vgl. "Material und Methoden") in einem Ablauf (A-D) und einer danebenliegenden, gleichzeitig untersuchten Pfütze $(E)$ von etwa $3 \mathrm{~m}^{2}$ Größe und $1-3 \mathrm{~cm}$ Tiefe mit dichtem Bewuchs von Zostera noltii. Die durchgehenden, grauen Säulen kennzeichnen die Zeitintervalle, in denen die Beobachtungsfläche durch Wolken beschattet war. Im Bereich von 10-90\% zeigen die Tiere im Ablauf (D) positive Rheotaxis, in der Pfütze (E) schreiten sie ungerichtet umher, Oberhalb von $90 \%$ aktiver $C$. crangon ist im Ablauf "Exodus" zu verzeichnen, in der Pfütze ungerichtetes Schwimmen 
wird vor allem durch Temperaturmessungen mit einem elektronischen Fühler (Tastotherm) gestützt: Sonneneinstrahlung von nur wenigen Minuten Dauer bewirkt eine Aufheizung der obersten Sedimentschichten in Abläufen und Pfützen. In 1-2 mm Tiefe liegen die Temperaturen 0,1 bis $0,6^{\circ} \mathrm{C}$ über der aktuellen Wassertemperatur. Dunkle Sedimente werden einige ${ }^{1 / 10}$ Grad stärker erwärmt als helle. Künstliche Beschattung resuliert in einer sofortigen Abkühlung der Sedimentoberfläche bis zu $0,5^{\circ} \mathrm{C}$ unterhalb der Wassertemperatur. Diese Tatsache erklärt in Abb. 3 den sprunghaften Rückgang der Gamelenaktivität bis hin zum Einschlagen in die obersten Sedimentschichten in genau dem Moment, von dem an die Beobachtungsfläche durch Wolken o. a. beschattet wird: Die Tiere suchen bei extremer Wärmeeinwirkung immer die am wenigsten erhitzten Bereiche auf. Entsprechendes gilt außer für Crangon crangon auch für die jungen Plattfische.

Die Auswirkungen eines massiven Exodus illustriert Abb. 4: Während mit der 2-mBaumkurre bei Hochwasser auf einem Untersuchungsabschnitt bei Station 7 senkrecht zum Hauptpriel vor einem Exodus ufernah noch große Mengen eben metamorphosierter Schollen gefangen werden konnten, fehlen sie nach dem Exodus in diesem Bereich fast völlig. Prielnah sind die Schollendichten hingegen angestiegen. Dieser außerordentlich

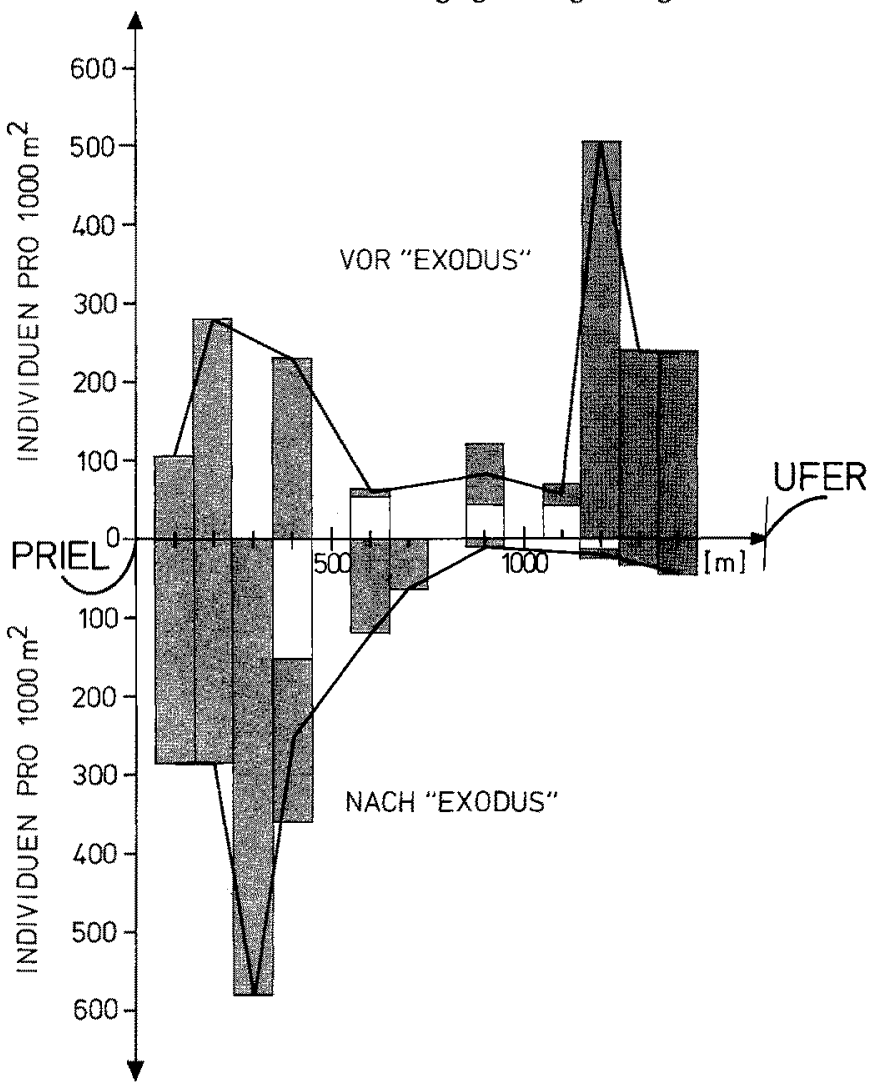

Abb. 4. Verteilung von Schollen der 0-Gruppe bei Hochwasser zwischen Hauptpriel und Ufer vor (24. Mai 1982) und nach einem "Exodus" (3. Juni 1982). Helle Säulen sind Doppelprobenergebnisse (mit Korrektur auf Netzselektion nach Kuipers, 1975) 
starke Exodus konnte am 27. Mai 1982 bei Niedrigwasser verzeichnet werden. Darüber hinaus ergaben Pfützenbefischungen mit dem Schiebehamen, daß nach dem Exodus vom 27. Mai an allen Stationen kaum noch junge Schollen und Flundern in den ufernăhen Pfützen ber-Niedrigwasser angetroffen werden konnten. Damit kann ein erneuter Exodus bei diesen Arten erst dann wieder auftreten, wenn neue Individuen in Ufernähe zum Bodenleben übergegangen sind, was bei Plattfischen in größerem Umfang oft erst im darauffolgenden Frühjahr der Fall ist. Ein starker Exodus bei Plattfischen ist daher ein im Jahresgang bei den einzelnen Arten meist einmaliges, für den Untersuchenden im Zeitpunkt schwer vorhersehbares Ereignis.

Synchron zu den Exodusvorgängen ist ein weiterer Verhaltenstypus im ArenicolaWatt zu beobachten: Die kleinen Garnelen, die auf den großen, 1-3 cm tiefen Pfützenbereichen weit verteilt sind, konzentrieren sich immer mehr in den Arenicola-Trichtern und schlagen sich dort ein. Dichten von 30 Tieren pro Trichter sind keine Seltenheit. Trocknet die Pfütze über einem solchen Trichter aus, findet man Crangon crangon z. T. in Tiefen von mehreren Zentimetern in den lockeren und feuchten Sedimentschichten des Trichters. Sie werden aktiv und kommen hervor, sobald das auflaufende Wasser den Trichter erreicht, um dann im Flutsaum bis $3 \mathrm{~cm}$ Wassertiefe dicht an dicht ein Stück weiter auf die höheren Platen zu wandern. Dieses Verhalten kann auch dadurch ausgelöst werden, daß der Trichter vom Beobachter mit Wasser gefüllt wird. Die Tiere zeigen sich dann sofort an der Sedimentoberfläche.

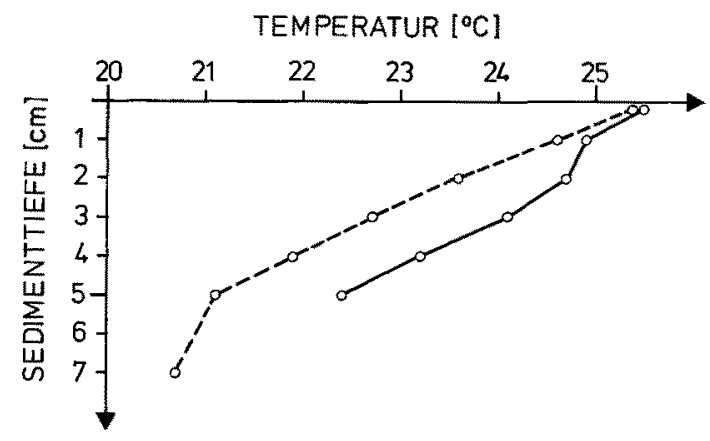

Abb. 5. Temperatur-Tiefen-Profile in einem trockengefallenen Arenicola marina-Trichter (-- - -) und im umgebenden, unüberstauten Sediment ( $\rightarrow$ vom 12. August 1982

Ein Vergleich der Temperatur-Tiefen-Profile für trockengefallene Arenicola-Trichter und für das umgebende, ebenfalls nicht überstaute Sediment in Abb. 5 macht deutlich, daß in den Trichtern auf den ersten 3 Zentimetern ein erheblich stärkerer Temperaturabfall mit der Tiefe zu verzeichnen ist. Von da an verlaufen beide Kurven parallel, der Arenicola-Trichter ist also immer $1-1,5^{\circ} \mathrm{C}$ kühler als das umgebende Sediment.

\section{DISKUSSION}

In Übereinstimmung mit Bergman et al. (1976) konnte im Bereich des Nordfriesischen Wattenmeeres festgestellt werden, daß Schollen in der ersten Zeit nach der Metamorphose noch keine Tidenwanderung von und zu den Prielen durchführen. Die Tiere konzentrieren sich vielmehr in den Pfützen und Abläufen auf den trockenfallen- 
den Platen. Entsprechendes gilt, wie die eigenen Beobachtungen zeigen, auch für die später im Jahr zum Bodenleben übergehenden Flundern und Seezungen. Damit wird deutlich, daß zumindest ein Teil dieser Plattfische nicht erst im Sommer in das Wattenmeer einwandert, wie früher vielfach angenommen (Creutzberg \& Fonds, 1971), sondern daß diese Tiere durch die sommerlichen Temperaturverhältnisse von den hohen Platen ins Sublitoral gedrängt und erst dort von den Untersuchungsschiffen gefangen werden. Daß Garnelen mit einer Länge von 2 bis $3,5 \mathrm{~cm}$ die Pfützen verlassen und zur Tidenwanderung übergehen, ist bereits das Ergebnis der Untersuchungen von Janssen \& Kuipers (1980). Nach Lillelund \& Berghahn (1981) sind die Längengruppen $\leqslant 2 \mathrm{~cm}$ im Nordfriesischen Wattenmeer bei Hochwasser in den hochgelegenen, ufernahen Bereichen sehr viel häufiger als auf den tieferliegenden Platen.

Im folgenden sollen 4 Fragenkomplexe diskutiert werden: (1) Welche Mechanismen bewirken die Konzentration der Tiere in den Pfützen? (2) Von welchen Faktoren wird das Verhalten der Tiere in den Pfützen und Abläufen beeinflußt? (3) Was veranlaßt die Tiere, die hohen Watten zu räumen? (4) Welche Vorteile bietet der Aufenthalt in den Pfützen, und welchen Gefahren sind die Tiere dabei ausgesetzt?

Ad (1): Creutzberg et al. (1978) konnten zeigen, daß metamorphosierende Schollen bei Hunger schwimmen und bei Sättigung zu Boden sinken. Die Autoren vergleichen in diesem Punkt das Wattenmeer mit seinem hohen Nahrungsangebot mit einer "Falle" für die Plattfische. Für das anschließende aktive Verbleiben der Postlarven von Schollen, Flundern, Seezungen und Garnelen in den Flachwassergebieten sowohl bei Hoch- als auch bei Niedrigwasser ist zum einen das Aufsuchen einer bestimmten Wassertiefe in Abhängigkeit von der Körpergröße (Wimpenny, 1960; Muus, 1967; Gibson, 1973; Janssen \& Kuipers, 1980), zum anderen die in der vorliegenden Untersuchung beschriebene positive Rheotaxis verantwortlich. Crangon crangon korrigiert seine Tiefeneinstellung nach Glas, Heyliges \& Beukema (unveröffentlicht, zitiert von Verwey, 1960) visuell über ein Anpeilen der Wasseroberfläche. Uber welche Rezeptoren Plattfische die Einhaltung bestimmter Wassertiefen kontrollieren, ist noch unklar. So reagieren Jungschollen kaum auf geringe Änderungen des hydrostatischen Drucks (Gibson, 1982). Dagegen konnte positive Rheotaxis bei $C$. crangon schon von Luther \& Maier (1963) bei Strömungsgeschwindigkeiten von 0,050 bis $0,058 \mathrm{~m} / \mathrm{s}$ experimentell nachgewiesen werden. Dahm (1973) konnte bis $0,02 \mathrm{~m} / \mathrm{s}$ eine derartige Reaktion nicht feststellen. Beide Befunde stützen folglich den in den Abläufen bestimmten Schwellenwert von etwa $0,05 \mathrm{~m} / \mathrm{s}$. Feldbeobachtungen zur positiven Rheotaxis bei Jungschollen machte Gibson (1973). Von Flundern ist allgemein bekannt, daß die Postlarven im Frühjahr von den Küsten die Flüsse stromauf wandern.

Ad (2): Schon Linke (1939) berichtet von Flundern und kleinen Garnelen in Wattenpfützen auf den Platen des Jadebusens und beschreibt, daß die Tiere bemüht sind, beim Trockenfallen die nächste tieferliegende Pfütze zu erreichen. Auch Kühl (1952) beobachtete junge $C$. crangon, die während des Tages in einer Pfütze schwammen, obwohl diese Art als nachtaktiv bekannt ist.

Die Ergebnisse der eigenen Arbeit zeigen, daß das Verhalten der Plattfische und Garnelen in den Pfützen und Abläufen bis hin zum Exodus mittelbar von der Wassertemperatur, aber unmittelbar von der Sonneneinstrahlung und damit den hohen Temperaturen der oberen Sedimentschichten beeinflußt wird. Die dunklen Sedimente in den Pfützen wirken wie die schwarze Platte in einem Sonnenkollektor, auf deren rasche 
Temperaturänderungen sich das überstehende Wasser nur träge anzupassen vermag. Der $\mathrm{O}_{2}$-Gehalt des Wassers, der stets über $4 \mathrm{ml} / 1$ (über $80 \%$ des Sättigungswertes) betrug unabhängig davon, ob ein Exodus zu verzeichnen war oder nicht, dürfte tagsüber die Aktivitätsänderungen und den Exodus nicht beeinflußt haben. Dies wird besonders durch die synchronen Beobachtungen und Messungen in einer Pfütze mit dichtem Bewuchs mit Zostera noltii und entsprechenden Sauerstoffübersättigungen belegt (Abb.3E). Abgesehen davon beträgt der kritische Sauerstoffgehalt $\left(\mathrm{LD}_{50}\right)$ für metamorphosierende Schollen $1,69 \mathrm{ml} / 1$ bei $8-13^{\circ} \mathrm{C}$ (De Silva \& Tytler, 1973), kleine C. crangon vermögen bei $21^{\circ} \mathrm{C}$ und einem Salzgehalt von $\mathrm{S}=23$ eine $\mathrm{O}_{2}$-Konzentration von $20 \%$ des Sättigungswertes $(1,16 \mathrm{ml} / \mathrm{l}) \mathrm{zu}$ überleben (Huddart \& Arthur, 1971). Salzgehalt-, $\mathrm{pH}$-Wert-, $\mathrm{NO}_{2}^{-}$- und $\mathrm{HS}^{-}$-Einflüsse scheiden in den beobachteten Fällen als Auslöser für die Verhaltensänderungen sowie die Aktivitätssteigerungen bis hin zur Flucht aus.

Die Fähigkeit der Tiere, bei Wassertemperaturen zwischen 20 . und $26^{\circ} \mathrm{C}$ auf Schwankungen von nur wenigen $1 / 10^{\circ} \mathrm{C}$ zu reagieren, erklärt das Verhaiten der Gartnelen in den Pfützen und Abläufen ebenso wie ihr Verhalten in den Arenicola-Trichtern: In den Trichtern ist oberflächennah ein stärkerer Temperaturabfall mit der Tiefe zu verzeichnen als auf den umgebenden Wattflächen. Diesem Gradienten können die Tiere folgen, wenn sie sich immer tiefer in das Sediment des austrocknenden Trichters einschlagen, was um so leichter ist, als die Partikel im Trichter lockerer gepackt sind. Bereits in einer Tiefe von $1 \mathrm{~cm}$ ist es im Trichter deutlich kühler als im benachbarten Sediment mit geringerem Wassergehalt, in $2 \mathrm{~cm}$ Tiefe beträgt die Differenz immerhin schon $1-1,5^{\circ} \mathrm{C}$. Arenicola-Trichter stellen somit bei Niedrigwasser ein wichtiges Refugium für kleine Garnelen dar.

Aquarienbeobachtungen über das Verhalten der vier in Rede stehenden Arten scheinen den dargelegten Befunden auf den ersten Blick zu widersprechen. Zahlreiche experimentelle Arbeiten über die Aktivitätsphasen von C. crangon (Hagerman, 1970; Dahm, 1973; Al-Adhub \& Naylor, 1975; Wienberg, 1976) berichten von einer ausgeprägten Nachtaktivität, die von einem Tidenrhythmus überlagert werden kann. In den wenigen Fällen, in denen dabei mit juvenilen $C$. crangon gearbeitet wurde, war aufgrund einer anderen Fragestellung nicht beabsichtigt, die Verhältnisse in den Pfützen - speziell die Erwärmung der oberen Sedimentschichten infolge der Sonneneinstrahlung - zu reproduzieren. Daher konnten keine vergleichbaren Aktivitäten der Tiere im Lichtversuch ohne Tidalrhythmik beobachtet werden (Dalley \& Bailey, 1981; van Donk \& de Wilde, 1981). Die Untersuchungen von Gibson et al. (1978) über die Aktivitätsphasen frisch metamorphosierter Schollen sind aus demselben Grund für die Situation in den Wattenpfützen nicht repräsentativ.

Ad (3): Es konnte gezeigt werden, daß in einem Wattengebiet mit langen Trockenfallzeiten bei Niedrigwasser Temperaturen an der Sedimentoberfläche und im überstehenden Wasser auftreten können, die postlarvale und juvenile Garnelen und Plattfische infolge der hohen physiologischen Beanspruchung zu einem fluchtartigen Verlassen dieses Habitats veranlassen, sofern ihnen eine Orientierungsmöglichkeit (Wasserströmung) geboten wird. Die Tiere zeigen mit ihrer Flucht zwei der drei von Kinne (1964) herausgestellten Reaktionen (Ausweichen, Kontaktverminderung, Akklimatisation) auf rasche Temperaturerhöhung. Laudien (1973) führt aus, daß diese Fluchtbewegungen nahe der Letaltemperatur sehr heftig sind. Die Temperaturen, bei denen der Exodus einsetzt, müssen demnach abhängig von der Akklimatisationstemperatur nahe der 
maximalen Hitzegrenze für die Tiere liegen. So ist nach Altman \& Dittmer (1966, zitiert nach Bush et al., 1972) die Letaltemperatur für $C$. crangon bei $30-32,5^{\circ} \mathrm{C}$ erreicht (Akklimatisationstemperatur $20^{\circ} \mathrm{C}$ ). Die im Rahmen des wasserchemisch-physikalischen Meßprogrammes vom 28. Juli 1982 gemessenen Temperaturwerte (Abb. 3 A) stiegen bis in diesen Bereich (Akklimatisationstemperatur, also aktuelle Temperatur des Prielwassers: $20,4-21,6^{\circ} \mathrm{C}$ ). Der gleichzeitig beobachtete Exodus ist demnach als eine Fluchtreaktion im Sinne von Laudien zu werten.

Am 15. Mai 1982 setzte ein Exodus von Schollen, Flundern und etwa $3 \mathrm{~cm}$ langen $C$. crangon bereits bei $27,2^{\circ} \mathrm{C}$ ein (aktuelle Temperatur an der Wasseroberfläche in den rielen: $12^{\circ} \mathrm{C}$ ) und erreichte bei $28^{\circ} \mathrm{C}$ seinen Höhepunkt. Für die Garnelen mag in diesem Fall die geringere Akklimatisationstemperatur gegenüber dem Sommer und/ oder die Tatsache eine Rolle gespielt haben, daß diese relativ großen Tiere eine geringere Toleranz für hohe Temperaturen haben. $27^{\circ} \mathrm{C}$ ist aber genau der Schwellenwert, bei dem nach Waede (1954) Jungschollen und -flundern aus der Ostsee von 5 bis $10 \mathrm{~cm}$ Länge "außerordentlich lebhaft" werden (Akklimatisationstemperatur $15^{\circ} \mathrm{C}$ ). Er konnte darüber hinaus zeigen, daß Schollen in Ostseewasser mit einem Salzgehalt von $\mathrm{S}=14-17$ bei Temperaturen von $28^{\circ} \mathrm{C} 4$ bis 5 Std. überleben, während Flundern eine solche Temperatur sogar $48 \mathrm{Std}$. überstehen. Ferner konnte für Schollen bei $\mathrm{S}=15$ und $28^{\circ} \mathrm{C}$ die Überlebensdauer durch vorherige Akklimatisation an $24^{\circ} \mathrm{C}$ erheblich verlängert werden. Ein vergleichbares Akklimatisationsphänomen war am 2. Juni 1982 bei postlarvalen Flundern gegenüber der bereits geschilderten Situation am 15. Mai zu beobachten: Bei einer Wassertemperatur von $28,0-29,6^{\circ} \mathrm{C}$ (aktuelle Oberflächentemperatur des Prielwassers: $17,8-18,3^{\circ} \mathrm{C}$ ) zeigten die Tiere positive Rheotaxis, aber noch kein Fluchtverhalten.

Die in den Pfützen und Abläufen ebenfalls häufig bis massenhaft vertretenen Corophien und die 1-10 mm großen Carcinus maenas sind am Exodus der Plattfische nicht beteiligt. Die Corophien ziehen sich in ihren Wohnröhren in kühlere Sedimentschichten zurück. Carcinus maenas ist wie Corophium volutator gegenüber hohen Temperaturen weitaus unempfindlicher als Crangon crangon (Altman \& Dittmer, 1966, zitiert nach Bush et al., 1972).

Der Begriff "Exodus" wird zusammenfassend definiert als eine in situ zu beobachtende, extreme, aber gerichtete Fluchtreaktion, die beim raschen Ansteigen der Umgeungstemperatur (oder der Veränderung anderer Faktoren) in letale Bereiche ausgelöst wird und großräumige Verteilungsänderungen bzw. das Aufsuchen anderer Habitate nach sich zieht. Ein starker Exodus kann dazu führen, daß die hohen Platen am Folgetag erheblich geringere Plattfischdichten aufweisen und zugleich die Dichten in Prielnähe stark ansteigen. Die beobachteten großräumigen Verteilungsverschiebungen bei Plattfischen müssen aber nicht immer auf einen einzigen, starken Exodus zurückzuführen sein. Vielmehr kann das Verlassen der ufernahen Bereiche abhängig von der Kontinuität und Stärke der Sonneneinstrahlung auch schub- oder etappenweise erfolgen. Verantwortlich dafür sind aber letztendlich die extremen Temperaturen, verbunden mit hohen tagesperiodischen Wassertemperaturschwankungen beim Einsetzen der Warmwetterperioden. Hinzu kommt das bereits im ersten Fragenkomplex angesprochene Phänomen, daß die genannten Arten mit zunehmender Länge größere Wassertiefen aufsuchen.

Erste Ergebnisse aus Untersuchungen an den Otolithen der Jungschollen deuten 
darauf hin, daß sich die hohe physiologische Beanspruchung der Tiere infolge der starken Temperaturfluktuationen und die hohe Aktivität vor und nach einem Exodus auf den Tagesringen (Panella, 1971) niederschlägt.

Ad (4): Die temperaturabhängige Aktivität der Garnelen bei Niedrigwasser und die damit verbundene Verlängerung der Nahrungsaufnahmephase dürfte den positiven Effekt noch verstärken, den eine Temperaturerhöhung auf das Wachstum der Juvenilen hat (Caudri, 1937; Meyer, zitiert nach Plagmann, 1937; Meixner, 1968). Entsprechendes gilt für die postlarvalen Plattfische in den Pfützen. Ferner stellt die zunächst weitgehende räumliche Trennung von aquatischen Freßfeinden einen Schutz für die jüngsten Stadien dar.

Vor dem Ubergang zur Tidenwanderung kann der Sterblichkeitsverlauf bei den 4 Arten von verschiedenen Faktoren beeinflußt werden. Neben dem stärkeren Feinddruck durch Vögel ist hier die von Zijlstra et al. (1982) vermutete dichteabhängige Sterblichkeit zu nennen. Ein Teil der Postlarven der Garnelen und der Seezungen dürfte den ab Ende Juni eines jeden Jahres massenhaft vorkommenden kleinen Carcinus maenas zum Opfer fallen. Wiederholt konnte beobachtet werden, wie stecknadelkopfgroße Strandkrabben die sehr viel schnelleren Garnelen zwischen 0,5 und 1,5 cm Länge in den Pfützen attackierten, sie dabei tödlich verletzten, um dann ihre reiche Beute zu verspeisen. Ferner können in Abläufen, in deren Einzugsbereich größere Zostera-Bestände oder dichte Diatomeenrasen liegen, im Laufe der Nacht empfindliche Sauerstoffdefizite auftreten (Broekhuysen, 1935). Was ein Trockenfallen der Tiere bei Niedrigwasser betrifft, so wird ihre Überlebenschance wesentlich davon abhängen, ob die Tiere ihre Kiemen feuchthalten können. So beträgt nach Mistakidis (zitiert nach Tiews, 1970) die Sterblichkeit für Crangon crangon nach einem 30minütigen Aufenthalt an der Luft bei $19^{\circ} \mathrm{C} 14-25 \%$. Wie eigene orientierende Vorversuche gezeigt haben, wird die Uberlebenschance stark erhöht, wenn sich die Tiere in die oberste, feuchte Sedimentschicht einschlagen können, und hängt neben der individuellen Konditionierung von folgenden Faktoren ab: (a) Sonneneinstrahlung (Dauer und Stärke), (b) Wassergehalt des Sediments (als Funktion von Sonne, Wind, Lufttemperatur, Höhenlage des Wattes etc.), (c) Korngröße des Sediments und (d) Verklebung des Sediments. Die Temperaturletalgrenzen für Plattfische und Garnelen dürften hier selten erreicht werden, weil Wattenflächen ohne Wasserüberstau infolge der Verdunstungskälte sich weniger erwärmen als vergleichbare Pfützensedimente. Während aber Ehrenbaum (1890) und Boddeke (1975) von Massensterben kleinster Crangon crangon durch die Wassertemperaturerhöhung in den Pfützen infolge starker Sonneneinstrahlung bei Niedrigwasser berichten, konnten in der vorliegenden Untersuchung nie tote juvenile $C$. crangon bei Niedrigwasser gefunden werden. Vielmehr überleben die auf den Platen bei Niedrigwasser zurückbleibenden kleinen Garnelen die in den Pfützen auftretenden Temperaturen von $30^{\circ} \mathrm{C}$ offenbar ohne nennenswerte Schäden (Tiews, 1970), wie auch in den Experimenten von van Donk \& de Wilde (1981) bestätigt werden konnte. Adulte Individuen von C. crangon reagieren demgegenüber sehr viel empfindlicher auf hohe Temperaturen (van Donk \& de Wilde, 1981): Schon ab $25^{\circ} \mathrm{C}$ ist eine Mortalität zu verzeichnen (Akklimatisationstemperatur vermutlich $20^{\circ} \mathrm{C}$ ).

Wenn Exodusvorgänge in den Abläufen an warmen, wolkenlosen Frühjahrs- und Sommertagen nicht durch Beschattung unterbrochen werden, kann es geschehen, daß viele der Plattfische durch die Einwirkung der hohen Temperaturen zugrunde gehen. 
Ein Teil der dann in den Abläufen schwimmenden Fische und Garnelen kann von Vögeln, insbesondere von Lachmöwen (Larus ridibundus) und von Silbermöwen (Larus argentatus) erbeutet werden. Für beide Arten stellen Garnelen und Fische wichtige Nährtiere dar (Goethe, 1980). Neuere Untersuchungen deuten darauf hin, daß eine hohe UV-Strahlung Fische schädigen kann (Bullock, 1982). Es bleibt zu prüfen, ob dieser Faktor gerade in den gutdurchlichteten Pfützen ein solches Gewicht bekommen kann, daß er die positiven Aspekte des Aufenthaltes in den Flachwasserbereichen aufhebt. Experimente zur Prüfung dieser Frage sowie zur Präzisierung der in den Vorversuchen gewonnenen Ergebnisse zum Trockenfallen der Tiere und zu ihrer Toleranz gegenüber hohen und tiefen Temperaturen sind in Vorbereitung. Dabei ist vorgesehen, die besonderen Bedingungen in den Pfützen zu simulieren.

Die Frage, welchen Einfluß Exodusvorgänge auf die Populationsdynamik der Juvenilen haben, kann erst mit Abschluß der Auswertung des in der Einleitung genannten Gesamtprogrammes zu beantworten versucht werden, weil in einer derartigen Bilanzierung eine große Zahl von Faktoren berücksichtigt werden muß. Die am Folgetag eines Exodus festgestellten $16 \%$ frisch verendeter Jungschollen in den prielnahen Fängen legen nahe, daß mit meßbaren Auswirkungen zu rechnen ist.

Danksagung. Ich danke Herrn Prof. Dr. K. Lillelund für seine Unterstützung bei dieser Arbeit, die Teil meiner Dissertation im Rahmen eines Projektes der Deutschen Forschungsgemeinschaft (DFG) ist (Li 93/31-1).

\section{ZITIERTE LITERATUR}

Al-Adhub, A. H. Y. \& Naylor, E., 1975. Emergence rhythms and tidal migrations in the brown shrimp Crangon crangon (L.). - J. mar. biol. Ass. U. K. 55, 801-810.

Bergman, M., Kuipers, B., Spliethoff, P. \& Veer, H. van der, 1976. Garnalen en krabben als mogelijke predatoren van 0-jarige schol op het balgzand. - Visserij 29, 432-438.

Boddeke, R., 1975. Autumn migration and vertical distribution of the brown shrimp Crangon crangon L. in relation to environmental conditions. In: Ninth European marine biology symposium. Ed. by H. Barnes. Aberdeen Univ. Press, Aberdeen, 483-494.

Broekhuysen, G. J. jr., 1935. The extremes in percentages of dissolved oxygen to which the fauna of a Zostera field in the tide zone at Nieuwediep can be exposed. - Archs néerl. Zool. 1, 339-346.

Bullock, A. M., 1982. The pathological effects of ultraviolet radiation on the epidermis of teleost fish with reference to the solar radiation effect in higher animals. - Proc. R. Soc. Edinb. (B) 81, 199-210.

Bush, R. M., Welch, E. B. \& Mar, B. W., 1972. Potential effects of thermal discharges on aquatic systems - basic data. Dep. Civil Eng. Univ. Washington, 47 pp.

Caudri, L. W. D., 1937. Einfluß der Temperatur und des Salzgehalts auf die Sterblichkeit von Garnelen (Crangon crangon L.). - Archs néerl. Zool. 3, 179-196.

Creutzberg, F. \& Fonds, M., 1971. The seasonal variation in the distribution of some demersal fish species in the Dutch Wadden Sea. - Thalassia jugosl. 7, 13-23.

Creutzberg, F., Eltink, A. Th. G. W. \& Noort, G. J. van, 1978. The migration of plaice larvae Pleuronectes platessa into the western Wadden Sea. In: Physiology and behaviour of marine organisms. Ed. by D. S. McLusky \& A. J. Berry. Pergamon Press, Oxford, 243-251.

Dahm, E., 1973. Untersuchungen zum Nahrungserwerb von Crangon crangon Linné. Diss., Univ. Hamburg, 81 pp.

Dalley, R. \& Bailey, H., 1981. A new apparatus used to record the locomotor rhythms of laboratory reared prawns and shrimps. - Mar. Ecol. Prog. Ser. 4, 229-234.

De Silva, C. D. \& Tytler, P., 1973. The influence of reduced environmental oxygen on the metabolism and survival of herring and plaice larvae. - Neth. J. Sea Res. 7, 345-362. 
Donk, E. van \& Wilde, P. A. W. J. de, 1981. Oxygen consumption and motile activity of the brown shrimp Crangon crangon related to temperature and body size. - Neth. J. Sea Res, 15, 54-64.

Ehrenbaum, E., 1890. Zur Naturgeschichte von Crangon vulgaris Fabr. - Studien über Bau, Entwicklung, Lebensweise und Fangverhältnisse des Nordsee-Granat. - Mitth. Sekt. KüstenHochseefisch. dt. FischVer, Sonderbeil., 1890, 1-124.

Gibson, R. N., 1973. The intertidal movements and distribution of young fish on a sandy beach with special reference to the plaice (Pleuronectes platessa L.). - J. exp. mar. Biol. Ecol. 12, 79-102.

Gibson, R. N., 1980. A quantitative description of the behaviour of wild juvenile plaice (Pleuronectes platessa L.). - Anim. Behav, 28, 1202-1216.

Gibson, R. N., 1982. The effect of hydrostatic pressure cycles on the activity of young plaice Pleuronectes platessa. - J. mar. biol. Ass. U. K. 62, 621-635.

Gibson, R. N., Blaxter, J. H. S. \& Groot, S. J. de, 1978. Developmental changes in the activity rhythms of the plaice (Pleuronectes platessa L.). In: Rhythmic activity of fishes. Ed. by J. E. Thorpe. Acad. Press, London, 169-186.

Goethe, F., 1980. Ecological data on bird species of the Wadden Sea - Black-headed gull - Herring gull. In: Birds of the Wadden Sea. Ed. by C. J. Smit \& W. J. Wolff. Balkema, Rotterdam, 219-229; 238-250. (Rep. Wadden Sea Working Group. 6.)

Groh, K., 1982. Die Besiedlung von Lahnungsfeldern in den Nordseewatten durch Jugendstadien von Crangon crangon (L.). - Dipl.-Arb., Univ. Hamburg, 111 pp.

Hagerman, L., 1970. Locomotory activity patterns of Crangon vulgaris (Fabricius) (Crustacea Natantia). - Ophelia 8, 255-266.

Huddart, R. \& Arthur, D. R., 1971. Shrimps in relation to oxygen depletion and its ecological significance in a polluted estuary, - Environ. Pollut. 2, 13-35.

Janssen, G. M. \& Kuipers, B. R., 1980. On tidal migration in the shrimp Crangon crangon. - Neth. J. Sea Res. 14, 339-348.

Kinne, O., 1964. Physiologische Aspekte des Lebens in Ästuarien. - Helgoländer wiss. Meeresunters. 11, 131-156.

Kühl, H., 1952. Über die Hydrographie von Wattenpfützen. - Helgoländer wiss, Meeresunters. 4, $101-106$.

Kuipers, B., 1975. On the efficiency of a two-meter beam trawl for juvenile plaice (Pleuronectes platessa). - Neth. J. Sea Res. 9,69-85.

Kuipers, B. R. \& Dapper, R. 1981. Production of Crangon crangon in the tidal zone of the Dutch Wadden Sea, - Neth. J. Sea Res. 15, 33-53.

Laudien, H., 1973. Activity, behavior etc. In: Temperature and life. Ed. by H. Precht, J, Christophersen, H. Hensel \& W. Larcher. Springer, Berlin, 441-501.

Lillelund, K. \& Berghahn, R., 1981. Gutachten zur Fischereibiologie der Nordstrander Bucht. In: Gutachten zur geplanten Vordeichung der Nordstrander Bucht. Hrsg. vom Minister für Ernährung, Landwirtschaft und Forsten des Landes Schleswig-Holstein. Rathmann, Raisdorf, $251-312$. (Schriftenreihe der Landesregierung Schleswig-Holstein. H. 12.)

Linke, O., 1939. Die Biota des Jadebusenwattes. - Helgoländer wiss. Meeresunters. 1, $201-348$.

Luther, W. \& Maier, H., 1963. Versuche über die Funktion der 1. Antenne von dekapoden Krebsen als Strömungssinnesorgan. - Helgoländer wiss. Meeresunters. 8, 321-332.

Meixner, R., 1968. Experimentelle Untersuchungen zur Biologie der Nordseegarnelen Crangon crangon (L.). - Diss., Univ. Hamburg, 102 pp.

Muus, B. J., 1967. The fauna of Danish estuaries and lagoons. - Meddr Danm. Fisk.-og Havunders. 5 (1), $1-316$.

Panella, G., 1971. Fish otoliths: daily growth layers and periodical patterns. - Science, N. Y. 173, 1124-1127.

Plagmann, J., 1937. Hydrographische Einflüsse auf die Lebensweise der Garnelen. - Fischmarkt 5 (2), $43-49$.

Rauck, G. \& Zijlstra, J. J., 1978. On the nursery aspects of the Wadden Sea for some commercial fish species and possible long-term changes. - Rapp. P.-v. Réun. Cons. perm. int. Explor. Mer 172 , $266-275$.

Riley, J. D., 1971. The Riley push-net. In: Methods for the study of marine benthos. Ed. by N. A. Holme \& A. D. McIntyre. Oxford, Blackwell, 286-290. 
Tiews, K., 1970. Synopsis of biological data on the common shrimp Crangon crangon (Linnaeus, 1758). - FAO Fish. Rep. 57 (4), 1167-1224.

Verwey, J., 1960. Über die Orientiertung wandernder Meerestiere. - Helgoländer wiss. Meeresunters. 7, 51-58.

Waede, M., 1954. Beobachtungen zur osmotischen und thermischen Resistenz der Scholle (Pleuronectes platessa) und Flunder (Pleuronectes flesus). - Kieler Meeresforsch. 10, 58-67.

Wienberg, M., 1976. Experimentelle Untersuchungen im infraroten Licht zur Bewegungsaktivität und Effektivität der Nahrungssuche der Nordseegarnele Crangon crangon. - Ber, dt. wiss. Kommn Meeresforsch. 24, 280-295.

Wimpenny, R. S., 1960. Young plaice hauls off the English east coast. - Fishery Invest., Lond. (Ser. 2) $23(1), 1-20$.

Zijlstra, J. J., Dapper, R. \& Witte, J. Ij., 1982. Settlement, growth and mortality of post-larval plaice (Pleuronectes platessa) in the western Wadden Sea. - Neth. J. Sea Res. 15, 250-272. 